\title{
VERIFICAÇÃO DO EFEITO AGUDO DA MOBILIZAÇÃO ARTICULAR NA FUNÇÃO PULMONAR DE INDIVIDUOS COM DOENÇA PULMONAR OBSTRUTIVA CRÔNICA (DPOC)
}

Bruno Paccini Alves Dócusse, Beatriz Anastácio Cipriano, Ygor Alberti Delício, Felipe Bezerra Santos, Flavio Danilo Mungo Pissulin, Carlos Eduardo Assunção de Freitas.

Universidade do Oeste Paulista - UNOESTE, Presidente Prudente, SP. E-mail: cfreitas@unoeste.br

\section{RESUMO}

A Doença Pulmonar Obstrutiva Crônica (DPOC) é caracterizada pela obstrução do fluxo aéreo e com consequência alteração na função pulmonar, comprometendo a força dos músculos respiratórios e a mobilidade da caixa torácica. A mobilização articular visa restabelecer a mobilidade e a artrocinemática das articulações da caixa torácica, entretanto, são escassos os estudos que investiguem a eficácia deste tipo de abordagem em pacientes com DPOC. O objetivo deste estudo foi avaliar os efeitos agudos da mobilização articular na função pulmonar de indivíduos com DPOC. A amostra foi composta por seis participantes com idade média de 56,33 $\pm 12,27$ anos, de ambos os sexos, portadores de DPOC. A função pulmonar foi avaliada por meio da espirometria, manuvacuometria e oximetria de pulso em dois momentos, antes e após o tratamento. A intervenção terapêutica foi constituída por técnicas de mobilização da coluna torácica, mobilização do esterno, e mobilização dos arcos costais respectivamente. Para a análise dos resultados foi utilizado o Teste Shapiro Wilk e o teste de T-Student pareado, considerando $p<0,05$. Observamos que não houve variações estatisticamente significativas após a aplicação das técnicas em nenhum dos parâmetros avaliados. Mais estudos são necessários para investigar os efeitos de técnicas mobilizatórias nesses pacientes.

Palavras-chave: DPOC, mobilidade torácica, osteopatia.

\section{VERIFICATION OF THE ACUTE EFFECT OF ARTICULAR MOBILIZATION ON THE PULMONARY FUNCTION OF INDIVIDUALS WITH CHRONIC OBSTRUCTIVE PULMONARY DISEASE (COPD)}

\begin{abstract}
Chronic Obstructive Pulmonary Disease (COPD) is characterized by airflow obstruction and consequent alteration in lung function, compromising respiratory muscle strength and rib cage mobility. Joint mobilization aims to restore arthrokinetic mobility of the rib cage joints; however, there are few studies investigating the efficacy of this approach in patients with COPD. The objective was to evaluate the acute effects of joint mobilization on pulmonary function in individuals with COPD. The sample consisted of six participants with a mean age of $56.33 \pm 12.27$ years of both sexes, with COPD. Pulmonary function was assessed by spirometry, manuvacuometry and pulse oximetry at two times, before and after treatment. The therapeutic intervention consisted of thoracic spine mobilization, sternal mobilization, and costal arch mobilization techniques, respectively. For the analysis of the results was used the Shapiro Wilk Test and paired Student $T$ test, considering $p<0.05$. It was observed that there were no statistically significant variations after the application of the techniques in any of the evaluated parameters. Further studies are needed to investigate the effects of mobilization techniques on these patients.
\end{abstract}

Keywords: COPD, thoracic mobility, osteopathy. 


\section{INTRODUÇÃO}

A Doença Pulmonar Obstrutiva Crônica (DPOC) atinge milhões de pessoas em todo o mundo, apresenta altas taxas de morbidade e já foi classificada como a terceira maior causa de morte no planeta ${ }^{1}$. Com as crescentes taxas de tabagismo e aumento da expectativa de vida estima-se um aumento nas taxas de prevalência nos países desenvolvidos ${ }^{2}$. No Brasil, a DPOC já foi a quinta maior causa de internação no Sistema Único de Saúde de pacientes com mais de 40 anos e responsável por aproximadamente 200.000 hospitalizações, gerando um gasto anual aproximado de 72 milhões de reais ${ }^{3}$.

A DPOC é uma patologia que não tem cura, porém tem tratamento, é caracterizada pela obstrução do fluxo aéreo de forma irreversível, representada no exame espirométrico por meio da relação entre o Volume Expiratório Forçada no 10 segundo $\left(\mathrm{VEF}_{1}\right)$ e a Capacidade Vital Forçada (CVF) ser menor que $0,7^{4}$. Além disso, compromete a força muscular dos músculos respiratórios e a mobilidade da caixa torácica que levam o indivíduo a ter uma maior dificuldade na realização de atividades físicas, e até mesmo atividades de vida diária 5 .

O tratamento indicado para esta doença consiste em grande parte na aplicação de programas de Reabilitação Cardiopulmonar (RCP) por meio do treinamento aeróbico e fortalecimento muscular ${ }^{6,7}$. A RCP possui alto grau de evidência científica quanto aos seus benefícios, sendo extremamente eficaz na melhora da tolerância ao exercício, redução da sensação de dispneia e melhora no índice de qualidade de vida relacionada à saúde. Entretanto, a mobilização passiva das articulações que compõem a caixa torácica não costuma ser incluídas nesse tipo de tratamento ${ }^{8}$.

A redução difusa da luz brônquica leva à hiperinsuflação pulmonar, bloqueando o tórax em inspiração. Este bloqueio, a longo prazo, gera retrações no tecido musculoesquelético da caixa torácica e que se tornam mais evidentes conforme ocorrem os períodos de exacerbação?. o diafragma se encontra retificado e consequentemente tem sua mobilidade diminuída trazendo consigo toda a cadeia conjuntiva que o suspende na base do crânio e na coluna vertebral até a quarta vértebra torácica, sendo este um fator importante para o aumento do ângulo de curvatura da cifose torácica que pode ser observado nesses pacientes e que está diretamente relacionada com a perda de mobilidade diafragmática ${ }^{10-14}$.

Tendo em vista estas alterações, outros métodos de tratamento, como a Osteopatia, e vêm sendo estudado visando melhorar a mobilidade torácica e funcionalidade dos músculos respiratórios para complementar o tratamento convencional ${ }^{15-17}$. A osteopatia é uma filosofia de tratamento que tem por finalidade restabelecer a harmonia entre o movimento dos tecidos, possibilitando que o corpo entre em homeostase ${ }^{18}$.

Estudos já foram realizados utilizando esses conceitos, mensurando as repercussões nos parâmetros respiratórios da aplicação de técnicas manuais nos componentes articulares e musculoesqueléticos da caixa torácica, tanto em pacientes saudáveis quanto em pneumopatas, porém, o tratamento se mostra mais eficiente na diminuição do Volume Residual quando realizado ao longo de um número maior de sessões, com uma conduta personalizada baseada na avaliação das disfunções de cada paciente e associada ao programa de reabilitação pulmonar ${ }^{19-21}$. Contudo, ainda são escassos os estudos que investiguem a eficácia da abordagem osteopática e de técnicas manuais em pacientes com DPOC, sendo assim, é de grande importância a realização de trabalhos nesta área, visando melhorar a qualidade das evidências que serão usadas como guia na prática clínica do fisioterapeuta. Dessa forma, o objetivo foi avaliar o efeito agudo da mobilização articular na função pulmonar de indivíduos com DPOC.

\section{METODOLOGIA}

Amostra

O estudo foi realizado num setor de reabilitação cardiopulmonar de uma universidade em Presidente Prudente, São Paulo. Foram avaliados seis indivíduos (amostra de conveniência) de ambos os sexos, com idade superior a 40 anos, com DPOC, identificados por diagnóstico clinico e exame espirométrico. Foram excluídos os indivíduos que apresentaram histórico de fratura recente nos arcos costais, esterno e coluna vertebral, os que sentiram dor ou desconforto durante a aplicação das técnicas e estiveram em um período de exacerbação da DPOC, aqueles que apresentaram pelo menor duas das seguintes alterações: aumento da dispneia, aumento da expectoração e aumento da purulência do escarro 22 . 
Aspectos de natureza ética

Este projeto de pesquisa foi submetido ao Comitê de Ética em Pesquisa (CEP) e obteve aprovação (CAAE 09546819.7.0000.5515) antes de seu inicio. Os participantes foram instruídos em relação a todos os procedimentos que seriam executados e após concordarem em participar da pesquisa assinaram o termo de consentimento livre e esclarecido.

\section{Desenho}

Trata-se de um estudo clínico experimental, transversal. Inicialmente foi realizada a coleta de dados antropométricos: idade, sexo, peso, altura, índice de massa corporal (IMC), profissão e uso de medicamentos.

A caracterização da amostra foi realizada a partir da análise dos prontuários dos participantes e realizada a espirometria para mensuração dos volumes pulmonares, seguida pela manovacuometria para obtenção da pressão expiratória máxima ${ }^{23}$. Após a avaliação inicial os participantes foram encaminhados para a realização da intervenção terapêutica e ao final da aplicação das técnicas foi feita a reavaliação utilizando novamente a espirometria e manovacuometria.

\section{Espirometria}

Todos os participantes fizeram uso do clipe nasal durante o exame, foram orientados a permanecerem sentados, a realizar uma inspiração profunda para atingir a capacidade pulmonar total. O tubo manteve-se próximo à boca e colocado nela imediatamente após o término da inspiração máxima, sobre a língua, entre os dentes e com os lábios cerrados para evitar vazamento de ar. A duração da expiração forçada foi de no mínimo 6 segundos com os indivíduos recebendo estímulo verbal durante a prova $^{23}$.

\section{Manovacuometria}

Os participantes do estudo fizeram uso do clipe nasal durante a utilização do manovacuômetro, onde foram orientados a permanecer sentados e introduzir o bucal na boca e realizar uma expiração com sua força máxima, e em seguida uma inspiração com sua força máxima onde não pode ter volume em suas bochechas. Foi considerado durante o teste $o$ valor que se manteve por maior tempo durante a expiração e a inspiração do voluntário. Também foram realizadas três aferições para a fase de pressão expiratória e três para a fase de pressão inspiratória na utilização do manovacuômetro ${ }^{23}$.

Intervenção terapêutica

Após a avaliação inicial os participantes repousaram por 5 minutos e receberam uma conduta composta pelas técnicas a seguir: A técnica de bombeio, realizada com o voluntário em decúbito dorsal, o terapeuta posicionado em finta anterior na lateral da maca voltado para a cabeça do paciente aplicando uma pressão em direção ao chão nas vértebras torácicas e orientando o paciente que deixe 0 ar sair pela boca durante as manobras ${ }^{24}$.

A mobilização dos arcos costais dos quadrantes superiores foi realizada com o paciente em decúbito dorsal, o terapeuta posicionado na ponta próximo à cabeça do paciente com as mãos posicionadas na porção superior em ambos os lados dos tórax. Foi solicitado que o voluntário realizasse uma inspiração e em seguida uma expiração lenta, durante a primeira expiração o terapeuta realizou uma pressão em direção ao chão e aos pés do paciente acompanhando o movimento dos arcos costais do quadrante superior, durante a inspiração o terapeuta manteve a resistência ofertada, tomando cuidado para não restringir em demasia a inspiração do paciente ${ }^{24}$.

A técnica de mobilização dos quadrantes inferiores foi realizada com o paciente em decúbito dorsal, o terapeuta posicionado de pé na ponta da maca próximo a cabeça do paciente com as mãos na parte lateral e inferior do tórax do voluntário. Foi solicitado que o voluntário realize uma inspiração e em seguida uma expiração lenta, durante a primeira expiração o terapeuta realiza uma pressão em direção póstero-caudal acompanhando o movimento dos arcos costais do quadrante inferior, durante a inspiração o terapeuta deve manter a resistência ofertada, tomando cuidado para não restringir em demasia a inspiração do paciente ${ }^{24}$.

A técnica de mobilização do esterno foi realizada com o paciente em decúbito dorsal, o terapeuta posicionado em pé lateralmente à maca com uma mão sobre o esterno reforçada pela outra mão, solicitou-se que o paciente realizasse uma inspiração profunda, durante a inspiração o terapeuta mobilizou o esterno em direção póstero-caudal, e durante a expiração manteve essa resistência ${ }^{24}$.

Terminada a conduta aplicada os participantes repousaram por 5 minutos e em 
seguida foi feita a reavaliação utilizando novamente a espirometria e manovacuometria.

Análise estatística

Os dados foram expressos por valores de média e desvio padrão (DP). O teste de normalidade Shapiro-Wilk foi inicialmente aplicado. Em seguida foi utilizado o teste estatístico T Student para comparação entre os grupos. Todas as análises foram realizadas no software Graphpad Prism, com nível de significância previamente estabelecido $(p<0,05)$.

\section{RESULTADOS}

Os resultados referentes à caracterização da amostra estão disponíveis na Tabela 1 . Os pacientes tinham média de idade de $56,33 \pm 12,27$ anos e todos foram classificados como DPOC grave $(G O L D=3)$ ou muito grave $(G O L D=4)$.
Tabela 1. Caracterização da amostra $(n=6)$.

\begin{tabular}{lc}
\hline Variáveis & Média \pm DP \\
\hline Idade (anos) & $56,33 \pm 12,27$ \\
Estatura $(\mathrm{m})$ & $1,57 \pm 0,07$ \\
Peso $(\mathrm{Kg})$ & $80,65 \pm 31,54$ \\
GOLD & $3 \pm 0,63$ \\
\hline
\end{tabular}

Os resultados das avaliações nos períodos pré e pós-intervenção estão descritos na Tabela 2 , onde nota-se que houve uma diminuição nos volumes captados pelo exame espirométrico no período pós intervenção, e um aumento na força muscular respiratória especialmente na pressão expiratória máxima (PEM) segundo os valores observados na manovacuometria, entretanto nenhum desses resultados pode ser considerado significativo pois não atingiram o valor pré estabelecido de $p<0,05$.

Tabela 2. Resultados das avaliações nos períodos pré e pós-intervenção (média \pm DP).

\begin{tabular}{lccc}
\hline Variáveis & Pré ( $\mathbf{n}=6)$ & Pós $(\mathbf{n}=6)$ & $\mathbf{P}$ \\
\hline CVF (L) & $1,89 \pm 0,5$ & $1,78 \pm 0,58$ & 0,7114 \\
CVF (\%) & $52,17 \pm 11,58$ & $49,50 \pm 12,66$ & 0,7384 \\
VEF1 (L) & $1,13 \pm 0,41$ & $1,13 \pm 0,45$ & 0,9896 \\
VEF1 (\%) & $27,65 \pm 52,68$ & $26,05 \pm 53,62$ & 0,9642 \\
PEM (mmHg) & $65(60-105)$ & $82(55-120)$ & 0,5844 \\
PIM (mmHg) & $55(40-120)$ & $57,5(45-120)$ & 0,6234 \\
\hline
\end{tabular}

Legenda: CVF - capacidade vital forçada, VEF1 - volume espirado após o primeiro segundo, VEF1/CVF - relação entre VEF1 e CVF, PEM - pressão expiratória máxima, PIM - pressão inspiratória máxima.

\section{DISCUSSÃO}

Apesar da escassez de estudos sobre o tema, é crescente na literatura a disponibilidade de estudos que investiguem a repercussão da mobilidade torácica em pacientes portadores de DPOC, assim como estudos que investiguem a eficácia da aplicação de técnicas de terapia manual em pneumopatas. Neste estudo podemos observar que as técnicas de mobilização articular não exerceram alterações agudas estatisticamente significativas, entretanto houve um aumento considerado da PEM e um ligeiro aumento da PIM. Tal aumento pode ser explicado pelo mecanismo descrito por Engel et al. ${ }^{25}$, que em revisão literária define o papel da TM como o de diminuir a rigidez torácica e devolver a funcionalidade e elasticidade dos músculos respiratórios, gerando alivio da sobrecarga exercida na musculatura acessória e redução dos sintomas em pacientes com DPOC.

Embora conflitantes, alguns trabalhos corroboram com nossos achados. Clarke et al. ${ }^{26}$ dizem que a abordagem manual tem resultados pouco expressivos a curto prazo, entretanto apresentam repercussões significativas se aplicados durante um número maior de sessões juntamente com programas de reabilitação cardiopulmonar. Já Galetti et al. ${ }^{27}$ concluiram em revisão sistemática que técnicas manuais associadas a RCP promovem melhora na capacidade funcional de exercício verificada pelo 
TC6 mas não promovem alterações na função pulmonar.

Buscemi et al. $^{28}$ verificaram o efeito da osteopatia em pacientes com DPOC moderado/grave, eles foram divididos em grupo $A$ (controle) o qual recebeu tratamento farmacológico e grupo $B$ (intervenção), que recebeu o mesmo tratamento mais o tratamento osteopático. Em sua intervenção foram abordados durante as sessões os nervos frênicos, seio maxilar, ligamentos vértebropleurais, costelas, pulmão, brônquios, músculos subclávios, ligamentos conóides e trapezoides. Apesar da diferença metodológica seus resultados corroboram com nosso trabalho e com estudos anteriores, uma vez que as alterações espirométricas não foram estatisticamente relevantes comparando os dois grupos, porém obtiveram melhora no CAT: intervenção $(p<0,0005)$ - controle $(p<0,188)$ e no TC6: intervenção $(p<0,0038)$ - controle $(p<0,5326)$.

Nos estudos em que houve variações significativas sobre parâmetros espirométricos podemos observar que houve ênfase na aplicação de técnicas de mobilização sobre tecidos moles soft tissue techniques (STT) durante várias sessões. Engel et al. $^{29}$ realizaram um estudo verificando o efeito a médio prazo da inclusão de técnicas manuais em programas de $R C P$, sua amostra foi dividida em três grupos sendo que um deles recebeu apenas a RCP, o segundo recebeu a RCP juntamente com STT, e por fim o terceiro recebeu as duas intervenções já citadas mais manipulações articulares na coluna vertebral. Após 24 semanas foi observado que o terceiro grupo apresentou um aumento de $0,4 \mathrm{~L}$ na $\operatorname{CVF}(p=0,03)$ quando comparado ao grupo que recebeu somente a RCP.

Dentre as STTs as técnicas de liberação miofascial aplicadas sobre o diafragma merecem menção especial, Rocha et al. ${ }^{30}$ avaliaram a repercussão da aplicação dessas técnicas sobre a capacidade inspiratória, mobilidade diafragmática, capacidade inspiratória, e capacidade funcional em pacientes com DPOC. Foram realizadas seis sessões utilizando as técnicas em dias não consecutivas ao longe de duas semanas, o grupo placebo recebeu intervenções falsas com a mesma periodicidade. Foram observados ganhos acumulativos ao longo das sessões na mobilidade diafragmática através da ultrassonografia com diferença de $18 \mathrm{~mm}$ entre os grupos, além de aumento na distância percorrida no TC6 com diferença de $22 \mathrm{~m}$ entre os grupos, a capacidade inspiratória estimada pela pletismografia optoeletronica também mostrou um aumento acumulativo de $330 \mathrm{~mL}$, e, como também foi mostrado em nossos resultados, houve um efeito agudo sobre pressão expiratória máxima porém não houve efeito acumulativo.

\section{CONCLUSÃO}

Os resultados obtidos em nosso trabalho permitiram observar que as técnicas de mobilização articular não exerceram efeito agudo sobre a função pulmonar em portadores de DPOC, entretanto o aumento dos parâmetros da manovacuometria podem indicar que as técnicas possam promover a diminuição momentânea da rigidez torácica. Entretanto diversos estudos mostram sua eficácia a médio e longo prazo associados a outras técnicas manuais e à RCP, significando que podem ser incluídas tais técnicas e abordagens no tratamento deste tipo de paciente como complemento ao tratamento convencional. Sugerimos que estudos futuros realizem um monitoramento a médio/longo prazo nesses indivíduos, e utilizem técnicas sobre tecidos moles associadas a técnicas articulares.

\section{DECLARAÇÃO DE CONFLITOS DE INTERESSE}

Os autores declaram não haver qualquer potencial conflito de interesse que possa interferir na imparcialidade deste trabalho científico.

\section{REFERÊNCIAS}

1. Lozano R, Naghavi $M$, Foreman $K$, Lim $S$, Shibuya K, Aboyans V, et al. Global and regional mortality from 235 causes of death for 20 age groups in 1990 and 2010: a systematic analysis for the Global Burden of Disease Study 2010. Lancet. 2012;380:2095-128. DOI: https://doi.org/10.1016/S0140-6736(12)61728-0

2. Global Initiative for Chronic Obstructive Lung Disease. Global strategy for the diagnosis, management, and prevention of chronic obstructive pulmonary disease; 2019 report. Michigan, USA. Eur Respir J. 2019;53(5):pii: $1900164 . \quad$ DOI: http://dx.doi.org/10.1183/13993003.00164-2019

3. Pessôa CLC, Pessôa RS. Epidemiologia da DPOC no presente - aspectos nacionais e internacionais. Pulmão RJ - Atualizações Temáticas. 2009;1(1):7-12. 
4. Kronborg T, Hangaard S, Cichosz SL, Hejlesen O. Increased accuracy after adjustment of spirometry threshold for diagnosing COPD based on pre-bronchodilator $\mathrm{FEV}_{1} / \mathrm{FVC}$. Respir Care. 2019;64(1):85-90.

DOI:

http://dx.doi.org/10.4187/respcare.06148

5. Costa BDS, Glória LM, Santos MSB, Sarges ESNF, Almeida CNS. Uso da liberação miofascial como técnica coadjuvante no tratamento de portador de doença pulmonar obstrutiva crônica. In: Congresso Internacional de Envelhecimento Humano. Acesso em 15 out 2018. Disponível em: http://www.cieh.com.br/

6. Maia EC, Pinheiro NA, Silva SCS, Ribeiro Filho FS, Navarro F, Oliveira Junior MNS. Protocolos clínicos de reabilitação pulmonar em pacientes com DPOC. Saúde Rev. 2012;12(32):55-67. https://doi.org/10.15600/2238-

1244/sr.v12n32p55-67

7. Kunikoshita LN, Silva YP, Silva TLP, Costa D, Jamami $M$. Efeitos de três programas de fisioterapia respiratória (PFR) em portadores de DPOC. Rev Bras Fisioter. 2006;10(4):449-55. https://doi.org/10.1590/S1413-

\section{$\underline{35552006000400014}$}

8. Spruit MA, Singh SJ, Garvey C, ZuWallack R, Nici $L$, Rochester $C$, et al. An official American Thoracic Society/European respiratory society statement: key concepts and advances in pulmonary rehabilitation. Am J Respir Crit Care Med. 2013;188(8):e13-e64. https://doi.org/10.1164/rccm.201309-1634ST

9. Lima PAL, Santana LSR. Alterações biomecânicas em portadores de doença pulmonar obstrutiva crônica. Ver FAFIBE online. Acesso em 15 out 2018. Disponível em: http://unifafibe.com.br/revistasonline/arquivos/r evistafafibeonline/sumario/16/30032011212258. pdf

10. Souza RMP, Cardim AB, Maia TO, Rocha LG, Bezerra SD, Marinho PEM. Inspiratory muscle strength, diaphragmatic mobility, and body composition in chronic obstructive pulmonary disease. Physiother Res Int. 2019;24(2):e1766. https://doi.org/10.1002/pri.1766
11. Souchard E. Respiração. 4ed. São Paulo: Summus Editorial; 1987. p.18.

12. Pachioni C, Ferrante J, Panissa T, Ferreira D, Ramos D, Moreira G, et al. Avaliação postural em pacientes com doença pulmonar obstrutiva crônica. Fisioter Pesq. 2011;18(4):341-5. https://doi.org/10.1590/S1809$\underline{29502011000400008}$

13. Gonçalves MA, Leal BE, Viegas GC, Lúcio MN, Mazo GZ, Paulin E. Relação entre a mobilidade diafragmática e as curvaturas da coluna vertebral em pacientes com doença pulmonar obstrutiva crônica. Fisioter Pesq. 2017;24(3):245-52. DOI: https://doi.org/10.1590/1809$\underline{2950 / 15809024032017}$

14. Gonçalves MA. Mobilidade diafragmática e cifose torácica em pacientes com doença pulmonar obstrutiva crônica. [Dissertação]. Florianópolis: Universidade do Estado de Santa Catarina; 2014.

15. Rodrigues CP, Matsuo T, Gonçalves CG, Hayashi D, Alves LA. Efeito de um programa de exercícios direcionados à mobilidade torácica na DPOC. Fisioter Mov. 2012;25(2):343-9. https://doi.org/10.1590/S0103-

\section{2}

16. Paulin E, Brunetto AF, Carvalho CRF. Efeitos de programa de exercícios físicos direcionado ao aumento da mobilidade torácica em pacientes portadores de doença pulmonar obstrutiva crônica. J Pneumol. 2003;29(5):287-94 . DOI: http://dx.doi.org/10.1590/S010235862003000500007

17. Moreno MA, Catai AM, Teodori RM, Borges BLA, Cesar MC, Silva E. Efeito de um programa de alongamento muscular pelo método de Reeducação Postural Global sobre a força muscular respiratória e a mobilidade toracoabdominal de homens jovens sedentários. J Bras Pneumol. 2007;36(6):679-86. DOI: $\quad$ http://dx.doi.org/10.1590/S1806$\underline{37132007000600011}$

18. Noll DR, Johnson JC, Baer RW, Snider EJ. The immediate effect of individual manipulation techniques on pulmonary function measures in persons with chronic obstructive pulmonary 
disease. Osteopath Med Prim Care. 2009;3(9):112. https://doi.org/10.1186/1750-4732-3-9

19. Santos JJA, Santos MCA, Carli J, Rocha P, Previatti KEK. Influência das técnicas de terapia manual osteopática na função respiratória. Arq Cienc Saúde UNIPAR. 2015;19(3):191-7. DOI: https://doi.org/10.25110/arqsaude.v19i3.2015.2 $\underline{873}$

20. Heneghan NR, Adab P, Balanos GM, Jordan RE. Manual therapy for chronic obstructive airways disease: A systematic review of current evidence. Manual Therapy. 2012;17:507-8. https://doi.org/10.1016/j.math.2012.05.004

21. Zanotti E, Berardinelli P, Bizzarri C, Civardi A, Manstretta A, Rossetti A, et al. Osteopathic manipulative treatment effectiveness in severe chronic obstructive pulmonary disease: A pilot study. Complement Ther Med. 2012;20(1-2):1622. https://doi.org/10.1016/i.ctim.2011.10.008

22. Fernandes FLA, Cukier $A$, Camelier AA, Fritscher CC, Costa $\mathrm{CH}$, Pereira EDB, et al. Recomendações para o tratamento farmacológico da DPOC: perguntas e respostas. J Bras Pneumol. 2017;43(4):290-301.

23. Shah SA, Velardo C, Farmer A, Tarassenko L. Exacerbations in chronic obstructive pulmonary disease: identification and prediction using a digital health system. J Med Internet Res. 2017;19(3):e69.

https://doi.org/10.2196/imir.7207

24. Ruiz FF. Cuadernos de osteopatia n.5. 3ed. Madrid: Dilema Editorial; 2007. p.98-135.

25. Engel $R$, Vemuplad $S$. The role of spinal manipulation, soft-tissue therapy, and exercise in chronic obstructive pulmonary disease: a review of the literature and proposal of an anatomical explanation. J Altern Complement Med. 2011;17(9):797-801.

https://doi.org/10.1089/acm.2010.0517

26. Clarke S, Munro EP, Lee LA. The role of manual therapy in patients with COPD. Healthcare (Basel). 2019;7(1):E21. https://doi.org/10.3390/healthcare7010021

27. Galletti J, Mcheileh G, Hahne A, Lee LA. The clinical effects of manipulative therapy in people with chronic obstructive pulmonary disease. J Altern Complement Med. 2018;24(7):677-83. https://doi.org/10.1089/acm.2017.0390

28. Buscemi A, Pennis V, Rapisarda A, Pennis A, Coco M. Efficacy of osteopathic treatment in patients with stable moderate-to-severe chronic obstructive pulmonary disease: a randomized controlled pilot study. J Altern Complement Med. 2019;17(1):20180128. https://doi.org/10.1515/jci $\underline{\mathrm{m}-2018-0128}$

29. Engel MR, Gonski $P$, Beath K, Vemuplad S. Medium term effects of including manual therapy in a pulmonar rehabilitation program for chronic obstructive pulmonary disease (COPD): a randomized controlled pilot trial. J Man Manip Ther. 201624(2):80-9. https://doi.org/10.1179/2042618614Y.00000000 $\underline{74}$

30. Rocha T, Souza H, Brandao CD, Rattes C, Ribeiro L, Campos LS, Aliverti A, Andrade DA. The manual diaphragm release technique improves diaphragmatic mobility, inspiratory capacity and exercise capacity in people with chronic obstructive pulmonary disease: a randomised trial. J Physiother. 2015;61(4):182-9. https://doi.org/10.1016/j.jphys.2015.08.009 